\title{
Compact compressive arc and beam switchyard for energy recovery linac-driven ultraviolet free electron lasers
}

\author{
J. A. G. Akkermans, ${ }^{1}$ S. Di Mitri, ${ }^{2, *}$ D. Douglas, ${ }^{3}$ and I. D. Setija ${ }^{1}$ \\ ${ }^{1}$ ASML Netherlands B.V., 5504 DR Veldhoven, Netherlands \\ ${ }^{2}$ Elettra Sincrotrone Trieste, 34149 Basovizza, Trieste, Italy \\ ${ }^{3}$ Thomas Jefferson National Accelerator Facility, Newport News, Virginia 23690, USA
}

(Received 24 March 2017; published 23 August 2017)

\begin{abstract}
High gain free electron lasers (FELs) driven by high repetition rate recirculating accelerators have received considerable attention in the scientific and industrial communities in recent years. Costperformance optimization of such facilities encourages limiting machine size and complexity, and a compact machine can be realized by combining bending and bunch length compression during the last stage of recirculation, just before lasing. The impact of coherent synchrotron radiation (CSR) on electron beam quality during compression can, however, limit FEL output power. When methods to counteract CSR are implemented, appropriate beam diagnostics become critical to ensure that the target beam parameters are met before lasing, as well as to guarantee reliable, predictable performance and rapid machine setup and recovery. This article describes a beam line for bunch compression and recirculation, and beam switchyard accessing a diagnostic line for EUV lasing at $1 \mathrm{GeV}$ beam energy. The footprint is modest, with $12 \mathrm{~m}$ compressive arc diameter and $\sim 20 \mathrm{~m}$ diagnostic line length. The design limits beam quality degradation due to CSR both in the compressor and in the switchyard. Advantages and drawbacks of two switchyard lines providing, respectively, off-line and on-line measurements are discussed. The entire design is scalable to different beam energies and charges.
\end{abstract}

DOI: $10.1103 /$ PhysRevAccelBeams.20.080705

\section{INTRODUCTION}

Soon after the advent of high gain free electron lasers (FELs) in the infrared (IR) and ultraviolet (UV) [1], scientific and industrial communities began considering the production of high average FEL power at tens of nm wavelength [2]. The production of multi-GW peak power level in UV and soft $x$ ray [3-5] is now well established at self-amplified spontaneous emission (SASE) [6] FEL facilities, and constitutes initial progress towards the implementation of $\mathrm{cw}$ or quasicontinuous (burst) lasing [7-10]. In fact, kW-level average power FELs at short wavelengths are anticipated within a few years [11,12].

In addition to high peak power and high pulse repetition rate, two other characteristics are required for cost-effective use in industrial applications such as EUV lithography [13]: system reliability and compactness. The following discussion addresses the issue of compactness, offering potential for unsurpassed performance in preserving electron beam brightness in a restricted machine footprint. An electron beam line design for the purpose of lasing in SASE mode at

\footnotetext{
*Corresponding author. simone.dimitri@elettra.eu

Published by the American Physical Society under the terms of the Creative Commons Attribution 4.0 International license. Further distribution of this work must maintain attribution to the author(s) and the published article's title, journal citation, and DOI.
}

the fundamental wavelength of $13.5 \mathrm{~nm}$ is presented. The accelerator is assumed to be a superconducting energyrecovery linac (ERL) in race-track geometry (Fig. 1). The electron beam is accelerated to the full energy of $1 \mathrm{GeV}$, and the bunch length compressed for reaching a bunch peak current of $\sim 1 \mathrm{kA}$ at the undulator entrance. It is subsequently decelerated for energy recovery and dumped.

In order to reduce machine size and complexity, bunch length compression in the compressive arc (CA) is performed during the very last stage of recirculation (Fig. 1). This scheme avoids any need for additional space in the layout for magnetic compressors, minimizes potential for beam quality degradation introduced by compression at lower energies, and maximizes available length for beam acceleration up to the undulator. It has, however, two drawbacks.

Firstly, in order to avoid degrading the FEL gain (and thus the output power for a given undulator length), the beam slice rms relative energy spread has to be smaller than the Pierce parameter, or energy-normalized FEL bandwidth. This is typically as small as $\sim 0.1 \%$ in EUV systems [14]. Consequently, the bunch linear energy chirp $h=$ $d E /(E d z)$ must be small in the CA, which in turn requires use of a large momentum compaction (or $R_{56}$ transport matrix element) [15] so as to achieve the desired compression factor $C=1 /\left|1+h R_{56}\right|$. A large $R_{56}$ implies in turn the presence of large energy dispersion in the arc dipole magnets, and thus introduces risk for emittance degradation in the bending plane through either chromatic aberrations or the CSR tail-head instability [16]. 


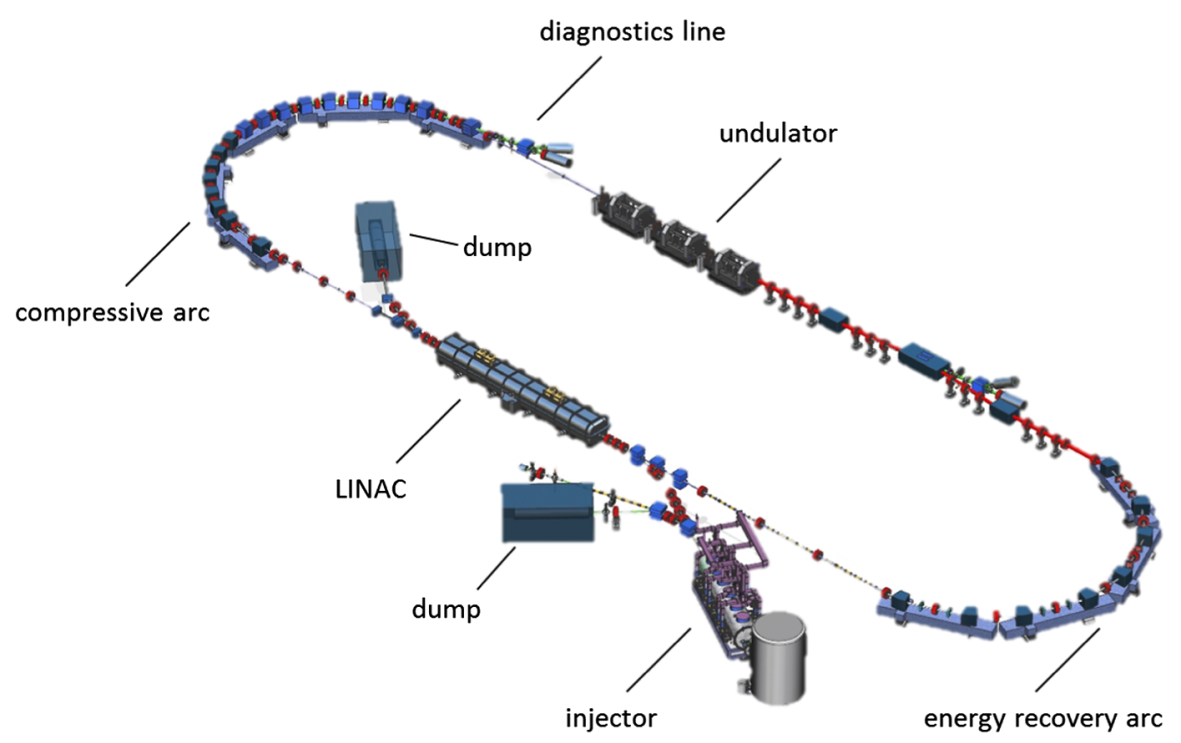

FIG. 1. Conceptual scheme of an ERL-FEL; beam moves clockwise. The scheme is not to scale, and a portion of the straight lines is omitted in order to make the arc lattice more evident.

Second, the CA has to be compact in order to allow a facility footprint of as small as a few tens of meters squared. However, a small average arc radius implies use of large individual dipole bend angles, resulting in increased impact of CSR effects on the projected emittance. The importance of minimizing projected emittance growth-while preserving bunch slice (local) emittance-is based in the need for short FEL gain length and for reliable transverse beam envelope matching to the undulator acceptance envelopes [17], both of which enhance FEL power while reducing required undulator length.

Accurate characterization of the beam six-dimensional (6D) phase space is needed to guarantee adequate control of beam parameters and to establish that a beam of the required quality is delivered to the undulator. This is especially important given that the bunch compression process can degrade the beam emittance. For this reason, electron beam diagnostic lines-complementing the design of the CA and located immediately after it - have been developed. Both off-line (operationally interruptive) and on-line (transparent to multibunch FEL operation) beam lines have been investigated; use of one or the other (or both) depends on the operational intent of the facility and on the tolerable beam quality degradation during transport.

The theoretical and numerical (simulation) design process for the compressive arc is presented in Sec. II. Though a presentation of a full accelerator design is beyond the scope of the present discussion, realistic beam parameters at the entrance of the compressor-consistent with the beam produced by a high repetition rate rf photoinjector [18] (Table I) - are considered. Preservation of the normalized beam emittance at the $0.1 \mu \mathrm{m} \mathrm{rad}$ level in either isochronous [19] or nonisochronous arcs [20] has, in principle, been demonstrated. However the performance of such beam lines has to date been inadequate for UV lasing when restricted to a compact footprint [21]. Here, instead, we generate optics cancellation of CSR energy kicks in a recirculating arc of $12 \mathrm{~m}$ of diameter and individual bend angle of $10 \mathrm{deg}$ (with commensurate suppression of emittance growth) by means of an analytical study and

TABLE I. Electron beam, compressive arc, and SASE FEL parameters. The electron distributions in the 6D phase space are all Gaussian with 2 sigma cutoffs.

\begin{tabular}{lcc}
\hline \hline Parameter & Value & Units \\
\hline Beam mean energy & 1 & $\mathrm{GeV}$ \\
Bunch charge & 100 & $\mathrm{pC}$ \\
Initial bunch length, rms & 0.6 & $\mathrm{~mm}$ \\
Initial peak current & 18 & $\mathrm{~A}$ \\
Initial total energy spread, rms & 0.1 & $\%$ \\
Initial energy chirp & -1.8 & $\mathrm{~m}^{-1}$ \\
Final peak current & 1 & $\mathrm{kA}$ \\
Total compression factor & 56 & \\
Arc $R_{56}$ & 0.53 & $\mathrm{~m}$ \\
Arc diameter & 12 & $\mathrm{~m}$ \\
Arc total bending angle & 180 & $\mathrm{deg}$ \\
Initial norm. projected emittance, & $0.5,0.5$ & $\mathrm{~mm} \mathrm{mrad}$ \\
$\quad$ rms (x,y) & $\leq 0.8, \leq 0.8$ & $\mathrm{~mm} \mathrm{mrad}$ \\
Final norm. projected emittance, & & \\
$\quad$ rms (x,y)-Tolerance & $\leq 0.6, \leq 0.6$ & $\mathrm{~mm} \mathrm{mrad}$ \\
Final norm. slice emittance, & & \\
$\quad$ rms (x,y)-Tolerance & 13.5 & $\mathrm{~nm}$ \\
FEL wavelength & 3 & \\
Undulator field parameter (K) & 0.13 & $\mathrm{~m}$ \\
Pierce parameter & 3.5 & $\mathrm{~m}$ \\
FEL gain length & 60 & \\
FEL saturation length & $\sim 1$ & $\mathrm{GW}$ \\
FEL peak power & & \\
\hline \hline
\end{tabular}



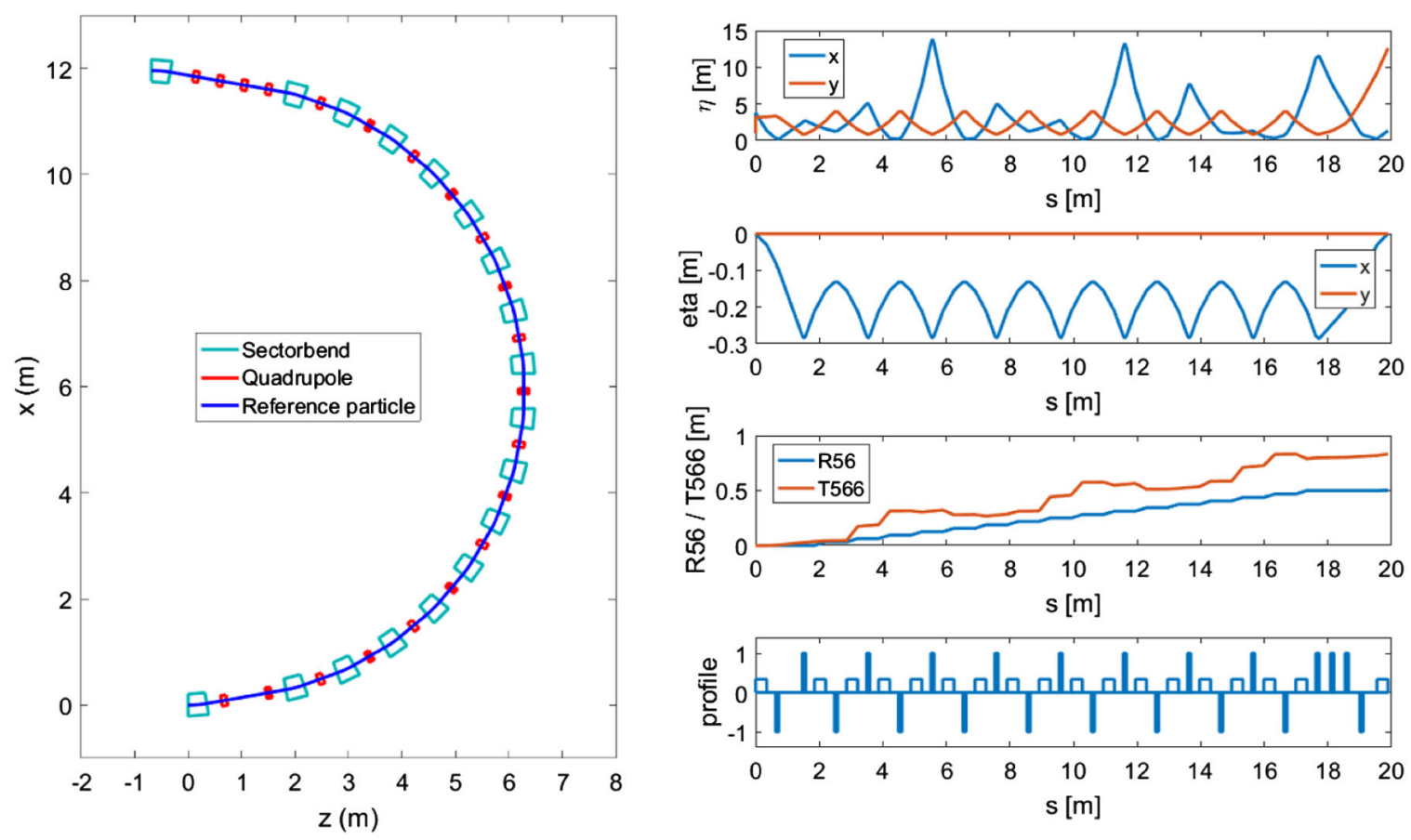

FIG. 2. Plan view of the FODO-arc lattice (left) and optics functions along it (from top to bottom, betatron functions, dispersion functions, first and second order transport matrix coefficients, magnetic elements).

modest numerical optimization. More specifically, a $100 \mathrm{pC}$ charge bunch is compressed in length by a factor $\sim 60$, reaching the final peak current of $1 \mathrm{kA}$ at an energy of $1 \mathrm{GeV}$. This electron beam brightness is consistent with requirements for GW-peak power single-pulse lasing, and for providing several tens of $\mathrm{kW}$-average FEL power from a $\mathrm{MHz}-\mathrm{level}$ bunch repetition rate superconducting linacthereby demonstrating the feasibility of an ERL-driven SASE FEL devoted to EUV lithography in a cost-effective and industrially compatible footprint.

The CA design is followed in Sec. III by a description of two diagnostic lines. At a length of $\sim 20 \mathrm{~m}$, these are compatible with the compact footprint of the facility. Although details of the diagnostics components are not the intent of this article, a list of diagnostic elements and their location along the line is given, and the capability of fast switching the compressed beam from one straight line (here, the FEL production line) to an off-axis line (the diagnostic line) is investigated. The lessons learned in this exercise may find application in FEL facilities servicing multiple users on fanned-out beam lines [22,23]. A discussion of the results and of the scaling of the beam lines design to different beam parameters is given in Sec. IV, where we also present conclusions.

\section{COMPRESSIVE ARC}

The single-stage compression scheme under consideration requires that the beam energy spread at the entrance of the CA be dominated by a linearly correlated energy chirp. This is assumed to have been applied to the beam in the linac upstream the arc (see Fig. 1). In order to address the most severe CSR effects-those occurring at the shortest bunch duration - we assume that the beam approaches the point of full compression (i.e. the longitudinal phase space is upright) at the arc exit. This scheme maximizes the peak current for any initial energy chirp; however, the rms uncorrelated relative energy spread has still to be smaller than the Pierce parameter. Table I summarizes the electron beam parameters, salient features of the compressive arc, and SASE FEL output performance as calculated using Xie's 3D formalism [24].

The arc magnetic lattice we consider is based on a regular pattern of alternating dipole magnets, Focusing and Defocusing quadrupole magnets (FODO), as shown in Fig. 2. The proposed arc includes 18 dipoles, each bending by $10 \mathrm{deg}$ at the beam energy of $1 \mathrm{GeV}$. The periodic ("matched") dispersion through each FODO cell is nonzero, and therefore dispersion matching sections ("dispersion suppressors") are needed at the start and the end of the arc to create and remove dispersion and to ensure that the periodic matching condition of individual arc cells is satisfied. Each dipole is $0.6 \mathrm{~m}$ long, the magnetic field is $1.0 \mathrm{~T}$, and the total $R_{56}=0.53 \mathrm{~m}$.

\section{A. CSR kick model}

The projected emittance growth induced by CSR in the bending plane is first estimated using the relatively simple model of 1D localized energy kicks in the linear optics approximation, and assuming steady-state CSR emission in dipole magnets [25]. To briefly review the physical 

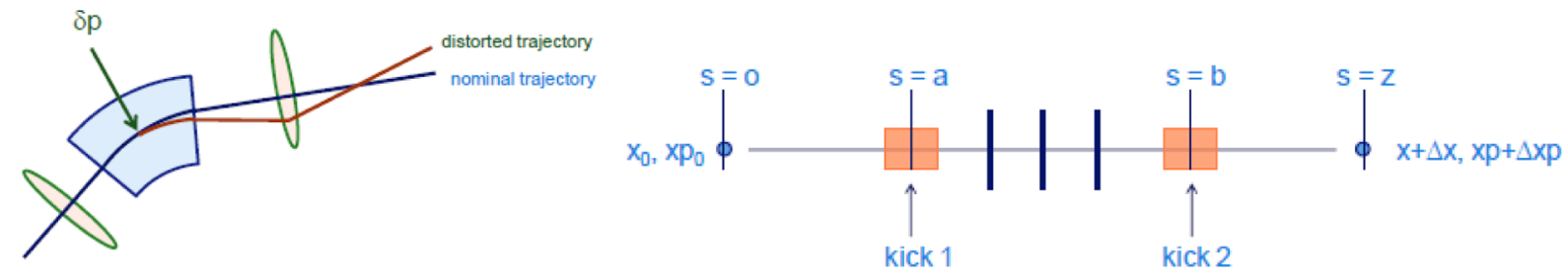

FIG. 3. Schematic of CSR kick model. Left: particle's trajectory is distorted by the local change of longitudinal momentum in a dispersive region, due to CSR emission. Right: double-bend achromatic cell used for comparison of ELEGANT result and analytical prediction of CSR-induced emittance growth, see Table II.

meaning of this model, we recall that the effect of CSR on an electron within a bunch can be approximated by an energy kick $\Delta E$ (or, equivalently, a longitudinal momentum kick $\delta \mathrm{p}_{z}$ ) that is applied within a dipole. The magnitude of the kick depends on the particle's longitudinal position within the bunch.

As a consequence of these energy shifts, each electron will start to deviate from the nominal dispersive trajectory (see diagram on the left of Fig. 3), betatron oscillating at an amplitude described by the momentum kick and the linear transport matrix from the position where the kick is applied to the end of the beam line [26,27] (see plot on right of Fig. 3). When evaluated at longitudinal coordinate $s$, the transverse position and angular divergence will respectively, deviate from the unperturbed trajectory by $\Delta x(s)=R_{16}^{a s} \delta p_{z}^{a} / p_{z, 0}+$ $R_{16}^{b s} \delta p_{z}^{b} / p_{z, 0} \quad$ and $\quad \Delta x^{\prime}(s)=R_{26}^{a s} \delta p_{z}^{a} / p_{z, 0}+R_{26}^{b s} \delta p_{z}^{b} / p_{z, 0}$. Here, $p_{z, 0}$ is the bunch mean longitudinal momentum, and CSR energy kicks are given at the location $s=a$ and $s=b$ along the particle trajectory. The change in particles' rms transverse coordinates at the end of the beam line leads to beam rms emittance growth, which can be estimated as [28] $\Delta \varepsilon_{x} \cong \varepsilon_{x, 0}\left(\beta_{x} \Delta x^{\prime 2}+2 \alpha_{x} \Delta x \Delta x^{\prime}+\gamma_{x} \Delta x^{2}\right)$. Here $\alpha, \beta, \gamma$ are the Twiss parameters at the end of the beam line, and $\Delta \mathrm{x}$, and $\Delta \mathrm{x}^{\prime}$ are now calculated as a function of the rms value of the CSR-induced relative energy deviation through the bunch (i.e., the relative energy spread). We recall that the CSR generated in a dipole decreases the mean energy of a Gaussian bunch by $\langle\Delta E\rangle \cong-0.35 \times e Q L_{b} /\left(4 \pi \varepsilon_{0} R^{2 / 3} \sigma_{z}^{4 / 3}\right)$ (in Standard System units) and the CSR-induced rms energy spread is $\sigma_{E} \cong 0.7 \times|\langle\Delta E\rangle|$ [29], where $Q$ is the bunch charge, $\mathrm{L}_{b}$ the dipole length, $R$ the dipole bending radius, and $\sigma_{z}$ the rms bunch length (which is assumed to be constant through the dipole).

This kick model can be used as the basis for a semianalytical approach to the description of the CSR head-tail instability. The CSR kick strengths at each dipole are evaluated analytically as a function of beam parameters and optics functions at that location. The resultant rms kick-and the corresponding emittance growth-are then calculated from the sum of the individual kicks. The accuracy of the kick model can be improved by taking multiple kicks per dipole into account (e.g., by numerically splitting the dipole), which is especially useful when the bunch length is varying considerably throughout the magnet.

For the purposes of this study, the kick model was benchmarked in the double-bend achromatic cell sketched in Fig. 3 (diagram on right) by comparison to emittance growth evaluated with particle tracking using ELEGANT [30], in the presence of bunch compression (the beam has an initial linear energy chip). Remarkable agreement for the final emittance is shown in Table II.

The kick model approach is valuable for a variety of reasons: (i) it is much faster than tracking a large number of particles, (ii) it can be used to evaluate the emittance growth for arbitrarily complex optics designs, including ones in which a fully analytical treatment [31-33] would be difficult, and (iii) it provides insight as to the contribution of each dipole to the final particle transverse displacement. We can therefore explore which dipoles are important for emittance growth and determine whether the transverse displacements introduced by different dipoles add up or cancel each other, as a function of the bunch length and of the Twiss parameters at each dipole's location.

Since the kick model is limited to the steady-state regime of CSR emission - and thus neglects the contribution of CSR effects due to propagation of radiation in drift sections downstream of magnets-it may in some cases underestimate the global CSR effect. In fact, particle tracking including CSR in drifts and in the transient regime at the dipoles' entrance $[25,29]$ indicates that a discrepancy of up to $25 \%$ in the final emittance value can arise at the shortest bunch duration considered here.

TABLE II. Analytical CSR kick model vs ELEGANT particle tracking prediction of projected emittance growth for the beam line sketched in Fig. 2. The initial bunch has $\mathrm{E}=1 \mathrm{GeV}$, $\mathrm{Q}=100 \mathrm{pC}, \mathrm{h}=10 \mathrm{~m}^{-1}, \varepsilon_{n, x}=0.5 \mu \mathrm{m} \mathrm{rad}$.

\begin{tabular}{lccc}
\hline \hline $\begin{array}{l}\text { Initial } \\
\text { bunch } \\
\text { length, } \\
\text { rms }[\mu \mathrm{m}]\end{array}$ & $\begin{array}{c}\text { Final bunch } \\
\text { length, } \\
\text { rms }[\mu \mathrm{m}]\end{array}$ & $\begin{array}{c}\text { Final projected } \\
\text { emittance from } \\
\text { kick model } \\
{[\mu \mathrm{m} \mathrm{rad}]}\end{array}$ & $\begin{array}{c}\text { Final projected } \\
\text { emittance } \\
\text { from tracking } \\
{[\mu \mathrm{m} \mathrm{rad}]}\end{array}$ \\
\hline 200 & 157 & 0.52 & 0.57 \\
100 & 79 & 0.69 & 0.74 \\
60 & 47 & 1.08 & 1.16 \\
30 & 24 & 2.46 & 2.49 \\
\hline \hline
\end{tabular}




\section{B. Beam envelope optimization}

Emittance growth in the presence of CSR kicks can be controlled either by reducing the absolute values of the particles' transverse displacements (e.g., by adopting small bending angles in relatively short dipole magnets) or, for a given lattice, by arranging the beam line optics in a way that kicks from distinct dipoles partially ("envelope optimization") [31] or fully cancel each other ("optics balance") [32]. Minimizing transport matrix terms associated with the dispersive motion-as required in the first scenario-is often not practical, since those terms are typically constrained by other requirements, including, for example, layout or footprint, compression factor, optics matching, etc. Envelope optimization requires massive particle tracking for a numerical optimization of the Twiss parameters at the line entrance in order to minimize the final emittance growth. A pure analytical optimization of the Twiss parameters using optics balance is impractical because of both the large number of elements included in the FODO arc and the asymmetry of the optics functions at the dipoles. For these reasons, the CSR-induced emittance growth through the arc was analyzed using the kick model as depicted above. The contribution of each dipole to the beam rms transverse displacement calculated at the end of the line is shown in the left plot of Fig. 4. From this result, we conclude that only the last three dipoles significantly contribute to emittance growth, presumably because the bunch is at its shortest length while traversing the final dipoles (see kick number 16-18). The kicks from these dipoles are directed in the same direction in the horizontal phase space (towards both negative $\mathrm{x}$ and negative $x^{\prime}$ ), and are separated from the other kicks, which are clustered around the origin, or (in the cases of kick number 13-15) directed toward positive $\mathrm{x}$ and $\mathrm{x}^{\prime}$. The latter difference offers potential for cancellation amongst the larger sets of kicks.

The final emittance was therefore minimized through a scan of the Twiss parameters at the arc entrance while keeping the arc quadrupole strengths fixed. The emittance growth was predicted analytically through the computation of the CSR kicks as a function of the Twiss parameters at the beginning of the line; the results are summarized in the right-hand plot of Fig. 4, which shows the projected normalized emittance growth vs final Twiss parameters. The solution shown in the right-hand plot of Fig. 2 and discussed below is for a choice of $\alpha_{x}=-2.5 \mathrm{rad}$ and $\beta_{x}=1.4 \mathrm{~m}$, which lies in the region of very low emittance growth (right-hand plot of Fig. 4) and also results in beam envelope functions-and beam sizes-that are small throughout the arc. For these initial conditions, the kick analysis predicts $\Delta \varepsilon_{n, x}=0.12 \mu \mathrm{m} \mathrm{rad}$, thereby satisfying the tolerance on emittance growth (Table I). The emittance growth predicted by a corresponding ELEGANT simulation with, and without, transient CSR effects is, respectively, $0.21 \mu \mathrm{m} \mathrm{rad}$ and $0.14 \mu \mathrm{mrad}$. Figure 5 shows the bunch current profile at the arc exit, and the projected normalized emittances along the arc. The simulation was performed using 10 million particles tracked with third order transport matrices, and includes incoherent and coherent synchrotron radiation.
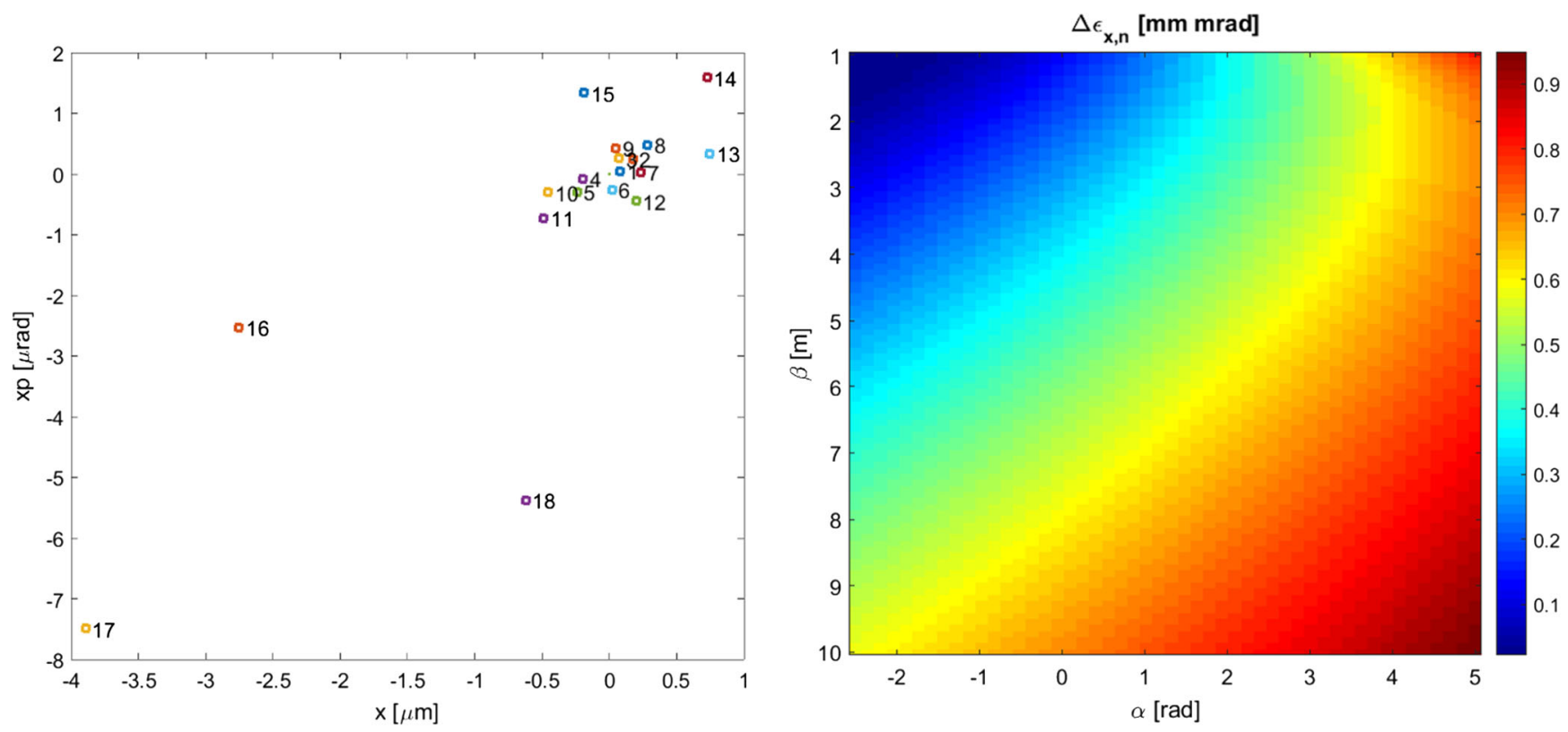

FIG. 4. Left: particle transverse displacement in the horizontal phase space due to CSR energy kicks in the FODO-compressive arc dipoles of Fig. 2. The numbers correspond to CSR kicks at the eighteen consecutive dipoles. Right: normalized projected horizontal emittance growth as a function of Twiss parameters at the end of the beam line, calculated with the CSR kick model. Compressive arc and beam parameters used for this study are listed in Table I. 

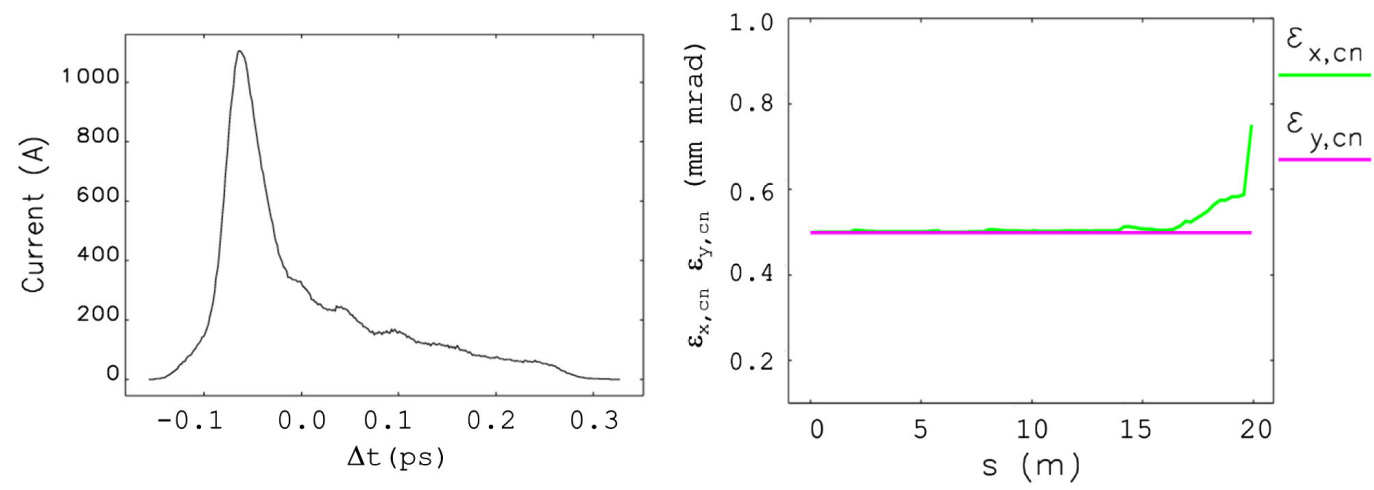

FIG. 5. Results from ElEGANT simulation for current profile at the exit of the CA (left), and projected normalized betatron emittances (right) along the CA. Slice emittances (not shown) are preserved at the injection level.

In this design, sextupole magnets are not included, as the natural evolution of nonlinearities in the longitudinal phase space (due to both higher order momentum compaction and CSR) assists in the generation of a current spike as desired and achieved (as shown in the left graphic of Fig. 5). Thus, systematic chromatic and geometric aberrations from higher order multipole magnets are avoided a priori. In general, numerical optimization of the magnetic lattice is also possible while using sextupoles, providing potential for cancellation of high order aberrations [33,34]. Performance might be further increased by employing, for example, a brute force genetic algorithm optimization [35]. It is, however, noteworthy that the emittance specifications of Table I can be met using simply the analytical approach.

\section{SWITCHYARD FOR DIAGNOSTIC LINES}

As discussed in the Introduction, diagnostic lines are to be installed between the CA and the undulator; we have developed designs that are parallel to the FEL production beam line. A maximum length of $\sim 30 \mathrm{~m}$ is allowed for a complete characterization of the $6 \mathrm{D}$ electron beam phase space using conventional diagnostic elements.

The design of a diagnostic line depends in part on the ERL beam filling/bunch timing pattern. Modes of operation in which beam-intercepting diagnostics can or cannot be used can be differentiated by distinguishing between high average beam power modes-which includes filling patterns that provide, e.g., $>100 \mathrm{~W}$ average beam power (corresponding to greater than $10 \%$ duty cycle at $>1 \mu \mathrm{A}$ average beam current) - and low average beam power modes-which includes all filling patterns with $\leq 100 \mathrm{~W}$ average beam power (corresponding to under $10 \%$ duty cycle with $\leq 1 \mu \mathrm{A}$ average beam current). We assume a maximum bunch repetition rate of $1 \mathrm{MHz}$ in cw. High average power options are associated with "production mode" (PM) scenarios; low average power options are referred to as "single bunch mode" (SM). In the PM, the beam must be stopped at dedicated dumps, and any intercepting or destructive measurement involving targets (e.g., screens) is prohibited in order to avoid damage. In contrast, during SM operation the beam can be lost in the vacuum chamber, and destructive measurements involving targets is allowed. In terms of diagnostics protection, any layout working in the PM will also work in the SM; the converse is obviously not true.

We first consider the beam line design for off-line diagnostics in SM. Then, the beam line supporting PM is presented. Technical specifications related to the functionality of the proposed diagnostics for different filling patterns (e.g., the dynamical range of sensors, resolution, etc.) are beyond the scope of this discussion.

\section{A. Horizontal branch}

A branch line can be connected to the penultimate dipole magnet of the CA, as shown in Fig. 6; this dipole is switched off when the beam has to be diagnosed. The last energized dipole in this configuration is followed by eight quadrupole magnets, which build up a $4 \times 4$ identity transport matrix for horizontal and vertical betatron motion. This is followed by an exact replica of the last double-bend cell of the CA, and then by the diagnostic line. Given the identical optics in the dipoles, the CSR effect in the last cell of the CA is reproduced at the entrance of the diagnostic line. The linear optics functions from the $\mathrm{CA}$ entrance to the end of the diagnostic line are shown in Fig. 7.

Conventional power converters supplying dc dipole magnets are not fast enough to switch from "off" to

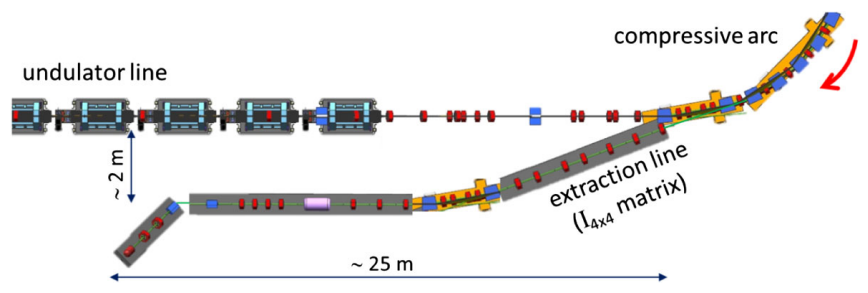

FIG. 6. Top view of the off-line diagnostic line as a branch of the CA. The beam moves from right to left. 


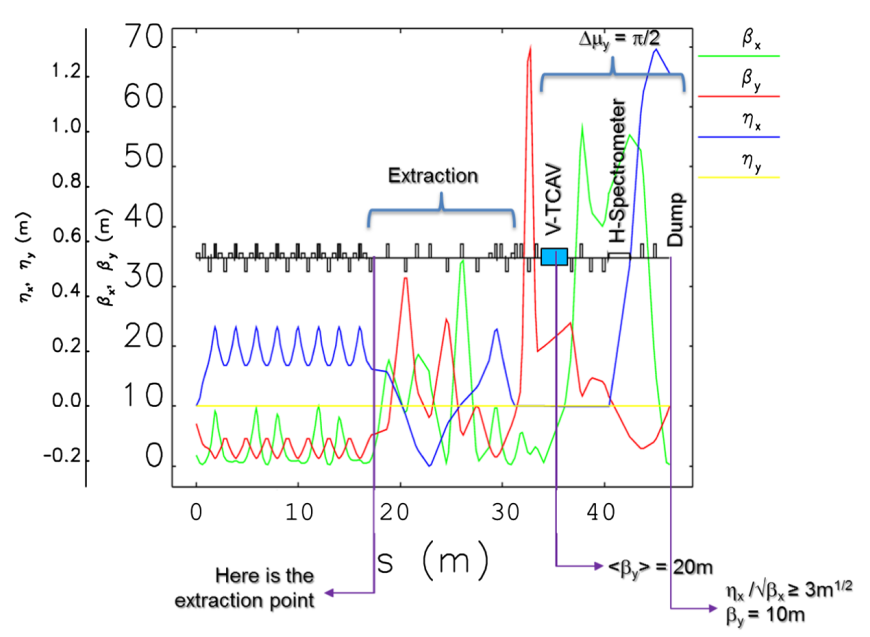

FIG. 7. Linear optics functions along the CA and the diagnostic branch line; the beam moves from left to right. The position of elements along the lattice and optics constraints for optimum diagnostic resolution is shown. The transport matrix for the horizontal and vertical betatron motion of the line portion, labeled "Extraction" in the figure, is the $4 \times 4$ identity matrix indicated in Fig. 6.

"on" states in a few hundred ns. It is therefore not possible to select individual bunches from a $\sim 1 \mathrm{MHz} \mathrm{cw}$ bunch train while directing the remainder to the FEL; consequently, this line can only be used for off-line measurements, interrupting FEL production. Given that intercepting diagnostics (such as screens) are to be used, the line must be utilized in SM. Conventional diagnostic elements included in the branch line for a full characterization of the electron beam are presented in some detail in Fig. 8.

\section{B. Vertical dogleg}

We now present a diagnostic line for on-line beam monitoring in PM. It is based on a method allowing a single "witness" bunch-injected into a short (e.g. $\sim 100 \mathrm{~ns}$ ) time window embedded in a $\sim 1 \mathrm{MHz}$ bunch filling pattern - to be kicked off from the production line and diagnosed. This is advantageous as it allows on-line monitoring of beam parameters at varying levels of average beam current. As the diagnostic line is off axis-and populated by only a single or few bunches at the desired repetition rate-intercepting diagnostics can be used.

Figure 8 shows a schematic of the line, in which the diagnostic transport branches off from the on-axis line devoted to FEL production. The witness bunch to be extracted from the cw bunch train is kicked horizontally by a fast stripline kicker $(\mathrm{K})$. A kicker rise and fall time shorter than $\sim 40 \mathrm{~ns}$ and a total kick angle of 5 mrad - at relatively low repetition rate (e.g. $\sim 100 \mathrm{~Hz}$ ) - are assumed at $1 \mathrm{GeV}$ [36,37]. After $2 \mathrm{~m}$, the kicked bunch enters a Lambertson septum (LS) at $10 \mathrm{~mm}$ lateral offset from the axis of the projection line; in the septum a horizontal magnetic field vertically bends the beam by $100 \mathrm{mrad}$ [38]. The same angle with opposite sign is provided by a second dc dipole (B) of the vertical dogleg. Optimization of the beam line design as to preserve the beam quality during extraction led to a modification of this initial layout, by expanding each dipole (LS, B in Fig. 8) in a double-bend cell ( $\mathrm{LS}+\mathrm{B}, \mathrm{B}+\mathrm{B})$. The optics functions of this final configuration are shown in Fig. 9, and the main parameters are listed in Table III.

Use of vertical extraction leaves horizontal motion unperturbed by CSR. Any residual CSR effect on the horizontal emittance occurring during compression-as

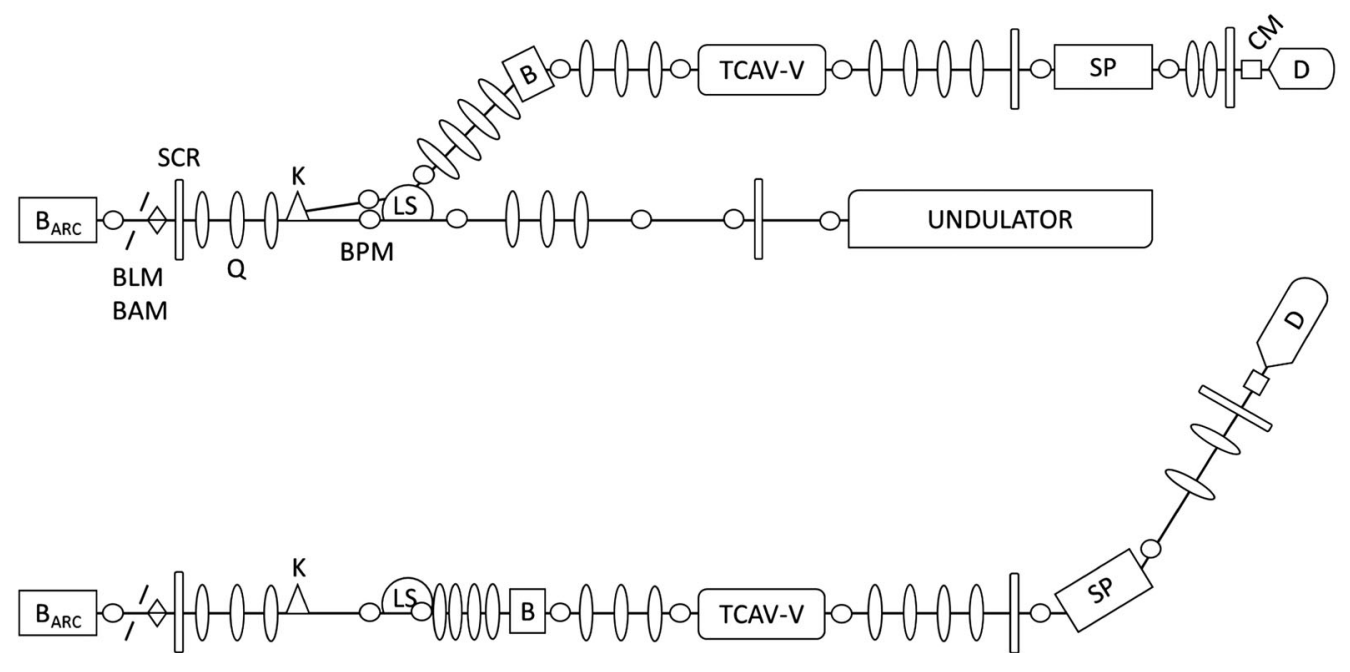

FIG. 8. Top: lateral view of the off-axis line, at the height of $1 \mathrm{~m}$ from the production line. The legend is as follows: $\mathrm{B}_{\mathrm{ARC}}=1$ last bending magnet of the compressive arc (dc dipole); $\mathrm{B}=$ bending magnet (dc dipole); $\mathrm{SP}=$ spectrometer (dc dipole); LS = Lambertson septum; $\mathrm{K}=$ fast kicker; $\mathrm{Q}=$ quadrupole magnet; $\mathrm{SCR}=$ screen system; $\mathrm{BPM}=$ beam position monitor; $\mathrm{TCAV}-\mathrm{V}=\mathrm{rf}$ transverse deflecting cavity, vertical; $\mathrm{BLM}=$ bunch length monitor; $\mathrm{BAM}=$ bunch arrival time monitor; $\mathrm{CM}=$ charge monitor; $\mathrm{D}=$ dump. Bottom: top view of the off-axis line. The beam moves from left (exit of CA) to right. Plots are not to scale. 


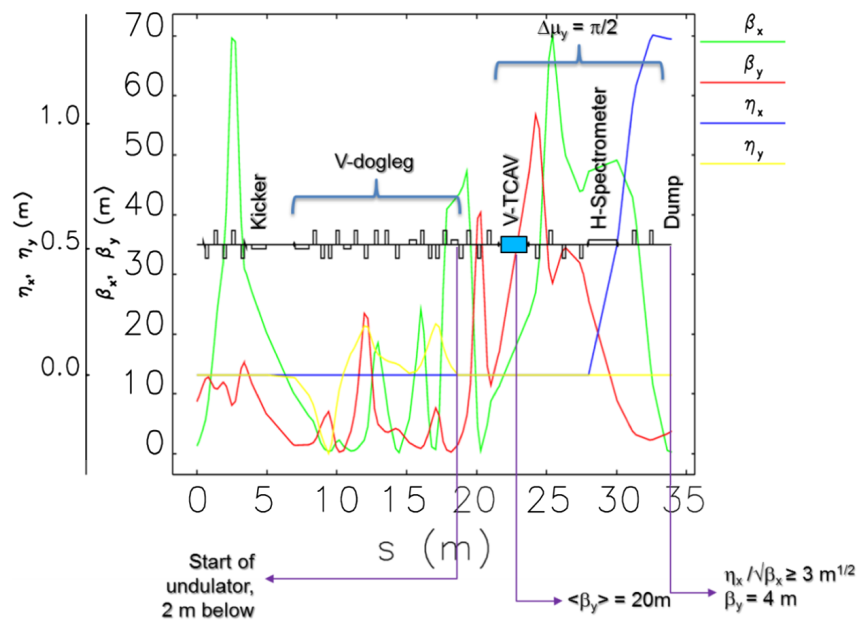

FIG. 9. Linear optics functions along the off-axis diagnostic line. Position of elements along the lattice and optics constraints for optimum diagnostic resolution are shown. The starting point is end of the CA, the beam moves from left to right.

well as the effectiveness of the optics tuning of the CA for CSR kick cancellation (see Sec. II) — can thus be accurately detected. Care is taken, however, to ensure CSR-induced vertical emittance growth is minimized through a suitable optics arrangement using several quadrupole magnets in the dogleg. They also ensure the line is achromatic and isochronous to first order: for a correlated fractional energy spread of $0.1 \% \mathrm{rms}$ and a bunch length of $\sim 20 \mu \mathrm{m} \mathrm{rms}$ (see Table I), we require $\left|R_{56}\right|<2 \mathrm{~mm}$. Quadrupole magnets in the off-axis line are used to adjust the extracted beam parameters to diagnostic design values, compensating the impact of the on-axis focusing quadrupoles (which are set to give proper PM beam matching at the FEL). Off-axis matching constraints include Twiss envelope values through the line and optimization of time and energy resolution at the screens of the spectrometer line [39,40]. The optics design in Fig. 9 is intended to preserve both beam horizontal emittance and bunch length through the dogleg, but at the expense of some residual vertical emittance growth (see Fig. 10, below, and Table IV).

TABLE III. Parameters of the vertical dogleg.

\begin{tabular}{lcc}
\hline \hline Parameter & Value & Units \\
\hline Beam energy & 1.0 & $\mathrm{GeV}$ \\
Number of dipole magnets (including septum) & 4 & \\
Dipole length & 1.0 & $\mathrm{~m}$ \\
Dipole Field & 0.22 & $\mathrm{~T}$ \\
Dipole bending angle & 65 & $\mathrm{mrad}$ \\
Total $R_{56}$ & $10^{-5}$ & $\mathrm{~m}$ \\
Total $T_{566}$ & $10^{-2}$ & $\mathrm{~m}$ \\
Elevation & 1 & $\mathrm{~m}$ \\
Longitudinal occupancy & 13 & $\mathrm{~m}$ \\
\hline \hline
\end{tabular}
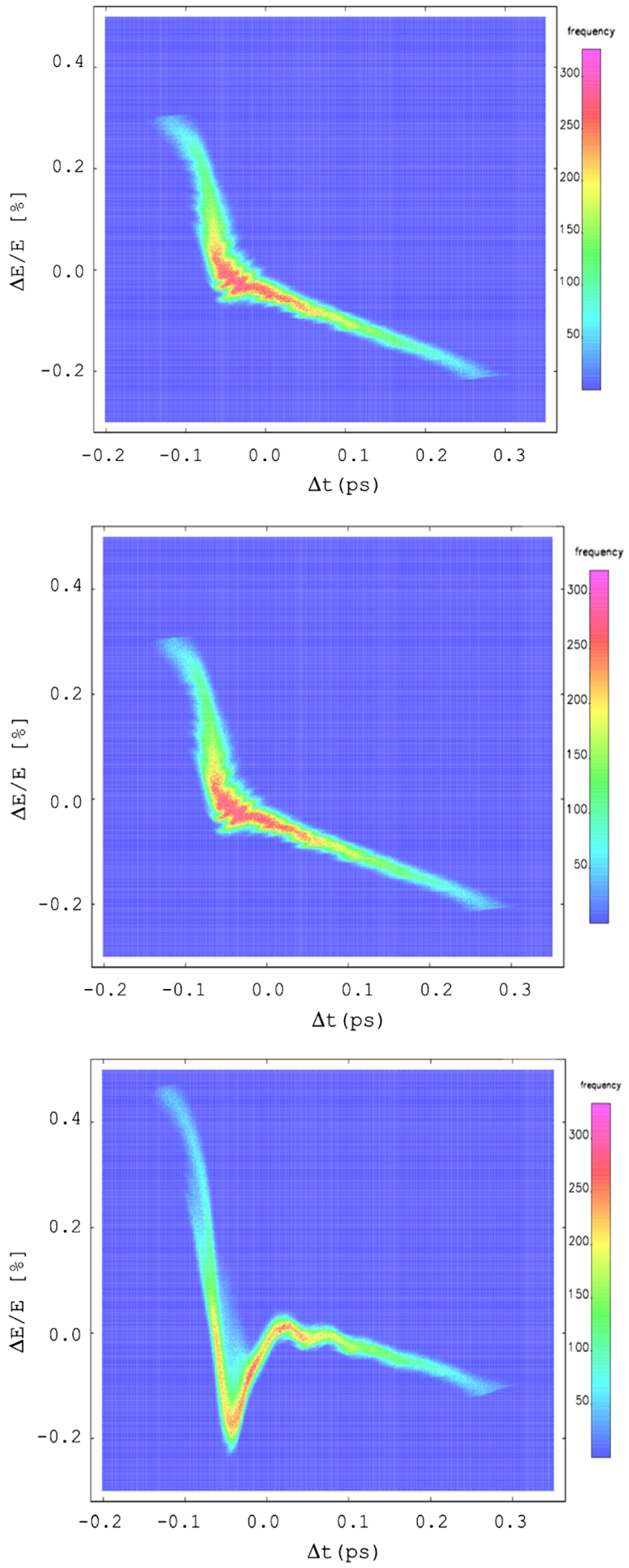

FIG. 10. From top to bottom: longitudinal phase space at the end of the CA (reference), at the end of the horizontal CA branch line, and at the exit of the vertical dogleg. 
TABLE IV. Beam parameters (all projected, rms values) at the exit of the CA, at the end of the horizontal branch line, and at the exit of the vertical dogleg.

\begin{tabular}{lcccc}
\hline \hline & $\begin{array}{c}\sigma_{z} \\
{[\mu \mathrm{m}]}\end{array}$ & $\begin{array}{c}\sigma_{\delta} \\
{[\%]}\end{array}$ & $\begin{array}{c}\varepsilon_{n, x} \\
{[\mu \mathrm{m} \mathrm{rad}]}\end{array}$ & $\begin{array}{c}\varepsilon_{n, y} \\
{[\mu \mathrm{m} \mathrm{rad}]}\end{array}$ \\
\hline End of CA (reference) & 26.0 & 0.106 & 0.71 & 0.50 \\
End of CA branch line & 26.3 & 0.107 & 0.74 & 0.50 \\
End of vertical dogleg & 26.2 & 0.109 & 0.76 & 0.71 \\
\hline \hline
\end{tabular}

\section{Comparison of switchyards}

Figure 10 compares the longitudinal phase space at the end of the CA, at the end of the horizontal CA branch line, and at the exit of the vertical dogleg, as obtained from ELEGANT simulation. Table IV summarizes the beam parameters at each location. While the vertical dogleg is suitable for on-line monitoring, it induces modifications in the longitudinal phase space-though without substantial changes in the macroscopic beam parameters - and vertical emittance growth. Both these effects are attributable to the CSR-induced energy spread in the dogleg. In contrast, the branch line provides fully transparent beam extraction from the production line, but at the price of off-line diagnostic capability in the present configuration and for high frequency $(1 \mathrm{MHz})$ bunch filling pattern.

\section{CONCLUSIONS}

A design for a $1 \mathrm{GeV}$ compressive arc, followed by a diagnostic line, in a compact footprint of $\sim 12 \times 20 \mathrm{~m}^{2}$ was presented; this study supports the realization of a compact, industrially suited EUV SASE FEL driven by an ERL. The arc design relies on an analytic model of the CSR tail-head instability, and offers performance (compactness, compression factor, peak current, emittance growth) surpassing that of prior art. Two diagnostic lines were also described; these can be used to verify horizontal emittance control in the compressive arc. The lines provide characterization of the single bunch $6 \mathrm{D}$ phase space using conventional diagnostics.

A horizontal branch line extending from the last cell of the compressive arc was shown to be transparent to the beam dynamics. However, it is not suited for on-line beam monitoring if the bunch repetition rate is high, as it cannot support intermittent extraction of bunches. This function could be provided, for example, were the penultimate cell of the arc designed with enough space in between the dipoles $(\sim 5 \mathrm{~m})$ to allow use of a fast stripline kicker and a suitable arrangement of quadrupole magnets so as to direct a witness beam to the diagnostic line. This solution is technically feasible, but requires a larger footprint than considered here. Cancellation of CSR kicks in such a design has yet to be demonstrated.

An alternative vertical dogleg design — preceded by fast beam extraction-does allow on-line beam monitoring during FEL operation. While the present configuration generates some perturbation in the beam energy distribution and vertical emittance due to CSR emission, these effects can be further minimized, and possibly made negligible, at the expense of either a lower bunch peak current or a longer beam line (by using smaller bending angles). The dogleg can, moreover, be made isochronous through second order by sextupole magnets-which would make the beam line even less invasive.

Due to the scaling of the CSR-induced modulation of relative energy offset with beam charge [16], CSR will have even less impact in both the arc and the diagnostic line designs at lower bunch charges (for similar bunch lengths) and higher beam energies. The vertical dogleg design provides guidance to the design of CSR-immune fast beam switchyards (such as those needed in facilities using large fan-out FEL beam lines) at full beam energy.

\section{ACKNOWLEDGMENTS}

The authors thank P. Smorenburg for helpful discussions about CSR modeling, and acknowledge support for this study from both the ASM Litography Holding N.V. company and the Industrial Liason Office of Elettra Sincrotrone Trieste.

[1] C. Pellegrini, The history of X-ray free-electron lasers, Eur. Phys. J. H 37, 659 (2012).

[2] C. Pagani, E. L. Saldin, E. A. Schneidmiller, and M. V. Yurkov, Design considerations of $10 \mathrm{~kW}$-scale extreme ultraviolet SASE FEL for lithography, Nucl. Instrum. Methods Phys. Res., Sect. A 463, 9 (2001).

[3] W. Ackermann, Operation of a free-electron laser from the extreme ultraviolet to the water window, Nat. Photonics 1, 336 (2007).

[4] P. Emma et al., First lasing and operation of an ångstromwavelength free-electron laser, Nat. Photonics 4, 641 (2010).

[5] T. Ishikawa et al., A compact X-ray free-electron laser emitting in the sub-ångström region, Nat. Photonics 6, 540 (2012).

[6] R. Bonifacio, C. Pellegrini, and L. Narducci, Collective instabilities and high-gain regime in a free electron laser, Opt. Commun. 50, 373 (1984).

[7] Y. Socol, G. N. Kulipanov, A. N. Matveenko, O. A. Shevchenko, and N.A. Vinokurov, Compact 13.5-nm free-electron laser for extreme ultraviolet lithography, Phys. Rev. ST Accel. Beams 14, 040702 (2011).

[8] P. H. Williams, D. Angal-Kalinin, D. J. Dunning, J. K. Jones, and N. R. Thompson, Recirculating linac freeelectron laser driver, Phys. Rev. ST Accel. Beams 14, 050704 (2011).

[9] S. V. Benson et al., A proposed VUV oscillator-based FEL upgrade at Jefferson Lab, J. Mod. Opt. 58, 1438 (2011).

[10] R. C. York, 5 upgradable to $25 \mathrm{keV}$ free electron laser facility, Phys. Rev. ST Accel. Beams 17, 010705 (2014). 
[11] M. Altarelli, The European X-ray Free-Electron Laser: toward an ultra-bright, high repetition-rate x-ray source, High Power Laser Sci. Eng. 3, e18 (2015).

[12] J. Stohr et al., SLAC National Accelerator Laboratory Report No. SLAC-R-978, 2011.

[13] E. A. Schneidmiller, V.F. Vogel, H. Weise, and M. V. Yurkov, J. Micro/Nanolithogr. MEMS MOEMS 11, 021122-1 (2012).

[14] P. Schmüser, M. Dohlus, and J. Rossbach, Ultraviolet and Soft X-ray Free Electron Lasers (Springer, Berlin, 2009), Vol. 229, ISBN: 978-3-540-79571-1.

[15] K. L. Brown, A first and second order matrix theory for the design of beam transport systems and charged particle spectrometers, Adv. Part. Phys. 1, 71 (1968).

[16] Ya. S. Derbenev, J. Rossbach, E. L. Saldin, and V. D. Shiltsev, TESLA-FEL 95-05 (DESY, Hamburg, Germany, 1995).

[17] S. Di Mitri and S. Spampinati, Estimate of free electron laser gain length in the presence of electron beam collective effects, Phys. Rev. ST Accel. Beams 17, 110702 (2014).

[18] A. Bartnik, C. Gulliford, I. Bazarov, L. Cultera, and B. Dunham, Operational experience with nanocoulomb bunch charges in the Cornell photoinjector, Phys. Rev. ST Accel. Beams 18, 083401 (2015).

[19] M. Venturini, Design of a triple-bend isochronous achromat with minimum coherent-synchrotron-radiation-induced emittance growth, Phys. Rev. Accel. Beams 19, 064401 (2016).

[20] S. Di Mitri and M. Cornacchia, Transverse emittancepreserving arc compressor for high-brightness electron beam-based light sources and colliders, Europhys. Lett. 109, 62002 (2015).

[21] N. Nakamura et al., in Proceedings of ERL 2015 (JacoW, Geneva, Switzerland, 2015), MOPCTH010.

[22] T. Hara et al., Pulse-by-pulse multi-beam-line operation for x-ray free-electron lasers, Phys. Rev. Accel. Beams 19, 020703 (2016).

[23] B. Faatz et al., Simultaneous operation of two soft x-ray free-electron lasers driven by one linear accelerator, New J. Phys. 18, 062002 (2016).

[24] M. Xie, in Proceedings of the Particle Accelerator Conference, Dallas, TX, 1995 (IEEE, New York, 1995), pp. 183-185.

[25] E. L. Saldin, E. A. Schneidmiller, and M. V. Yurkov, On the coherent radiation of an electron bunch moving in an arc of a circle, Nucl. Instrum. Methods Phys. Res., Sect. A 398, 373 (1997).
[26] R. Hajima, A first-order matrix approach to the analysis of electron beam emittance growth caused by coherent synchrotron radiation, Jpn. J. Appl. Phys. 42, L974 (2003).

[27] Y. Jiao, X. Cui, X. Huang, and G. Xu, Generic conditions for suppressing the coherent synchrotron radiation induced emittance growth in a two-dipole achromat, Phys. Rev. ST Accel. Beams 17, 060701 (2014).

[28] M. Venturini, CSR-induced emittance growth in achromats: Linear formalism revisited, Nucl. Instrum. Methods Phys. Res., Sect. A 794, 109 (2015).

[29] M. Borland, Simple method for particle tracking with coherent synchrotron radiation, Phys. Rev. ST Accel. Beams 4, 070701 (2001).

[30] M. Borland, APS Tech Note No. LS-207, 2000.

[31] R. Hajima, Emittance compensation in a return arc of an energy-recovery linac, Nucl. Instrum. Methods Phys. Res., Sect. A 528, 335 (2004).

[32] S. Di Mitri, M. Cornacchia, and S. Spampinati, Cancellation of Coherent Synchrotron Radiation Kicks with Optics Balance, Phys. Rev. Lett. 110, 014801 (2013).

[33] S. Di Mitri, Feasibility study of a periodic arc compressor in the presence of coherent synchrotron radiation, Nucl. Instrum. Methods Phys. Res., Sect. A 806, 184 (2016).

[34] P. Piot, D. R. Douglas, and G. A. Krafft, Longitudinal phase space manipulation in energy recovering linacdriven free-electron lasers, Phys. Rev. ST Accel. Beams 6, 030702 (2003).

[35] R. Bartolini, M. Apollonio, and I. P. S. Martin, Multiobjective genetic algorithm optimization of the beam dynamics in linac drivers for free electron lasers, Phys. Rev. ST Accel. Beams 15, 030701 (2012).

[36] C. Pappas et al., in Proceedings of the 5th International Particle Accelerator Conference, Dresden, Germany (JACoW, Geneva, Switzerland, 2014), MOPME083.

[37] M. Placidi, J.-Y. Jung, A. Ratti, and C. Sun, Compact spreader schemes, Nucl. Instrum. Methods Phys. Res., Sect. A 768, 14 (2014).

[38] D. Alesini, S. Guiducci, F. Marcellini, and P. Raimondi, Design, test, and operation of new tapered stripline injection kickers for the $e^{+} e^{-}$collider DAФNE, Phys. Rev. ST Accel. Beams 13, 111002 (2010).

[39] M. Röhrs, C. Gerth, H. Schlarb, B. Schmidt, and P. Schmüser, Time-resolved electron beam phase space tomography at a soft x-ray free-electron laser, Phys. Rev. ST Accel. Beams 12, 050704 (2009).

[40] S. Di Mitri and M. Cornacchia, in Proceedings of the 32nd Free Electron Laser Conference, Malmö, Sweden (Max-lab, Sweden, 2010), WEPB42. 